\title{
Strong chiral dichroism and enantiopurification in above-threshold ionization with locally chiral light
}

\author{
Ofer Neufeld $\odot,{ }^{1,2, *}$ Hannes Hübener $\odot,{ }^{1}$ Angel Rubio $\odot,{ }^{1,3}$ and Umberto De Giovannini $\odot{ }^{1,4}$ \\ ${ }^{1}$ Max Planck Institute for the Structure and Dynamics of Matter, Hamburg 22761, Germany \\ ${ }^{2}$ Physics Department and Solid State Institute, Technion-Israel Institute of Technology, Haifa 32000, Israel \\ ${ }^{3}$ Center for Computational Quantum Physics (CCQ), The Flatiron Institute, New York, New York 10010, USA \\ ${ }^{4}$ IKERBASQUE, Basque Foundation for Science, E-48011 Bilbao, Spain
}

(Received 2 October 2020; accepted 11 June 2021; published 6 July 2021)

\begin{abstract}
We derive here a highly selective photoelectron-based chirality-sensing technique that utilizes "locally chiral" laser pulses. We show that this approach results in strong chiral discrimination, where the standard forwards/backwards asymmetry of photoelectron circular dichroism (PECD) is lifted. The resulting dichroism is larger and more robust than conventional PECD (especially in the high-energy part of the spectrum), is found in all hemispheres, and is not symmetric or antisymmetric with respect to any symmetry operator. Remarkably, chiral dichroism of up to $10 \%$ survives in the angularly integrated above-threshold ionization (ATI) spectra, and chiral dichroism of up to $5 \%$ survives in the total ionization rates. We demonstrate these results through $a b$ initio calculations in the chiral molecules bromochlorofluoromethane, limonene, fenchone, and camphor. We also explore the parameter space of the locally chiral field and show that the observed dichroism is strongly correlated to the degree of chirality of the light, validating it as a measure for chiral-interaction strengths. Our results pave the way for highly selective probing of ultrafast chirality in ATI and motivate the use of locally chiral light for enhancing ultrafast spectroscopies. Most importantly, the technique can be implemented to achieve all-optical enantiopurification of chiral samples.
\end{abstract}

DOI: 10.1103/PhysRevResearch.3.L032006

Chirality is a ubiquitous naturally occurring phenomenon that plays a major role in Physics, Chemistry, and Biology. Its analysis, characterization, and manipulation are crucial both from a fundamental scientific point of view (e.g., for analyzing dynamical chemical processes [1], particle physics [2], and materials topology [3,4]), and from a practical aspect (e.g., in drug design [5]). In terms of analysis, chirality is standardly probed with chiroptical techniques that measure the response of the medium to optical excitations. Usually, these rely on absorption circular dichroism, which requires magnetic-dipolar and/or electric-quadrupolar interactions; hence this leads to very weak responses [6]. Several breakthroughs in the last decades have advanced methods that rely solely on electric-dipole interactions, and are accordingly much more efficient. These include perturbative second-order nonlinear effects [6-11], Coulomb explosion imaging [12,13], enantiospecific state transfer [14], photoelectron circular dichroism (PECD) in the single-photon [15-18] and multiphoton regimes [19-23] (including bichromatic collinear lasers [24,25]), photoion circular dichroism [26],

\footnotetext{
*ofer.neufeld@gmail.com
}

Published by the American Physical Society under the terms of the Creative Commons Attribution 4.0 International license. Further distribution of this work must maintain attribution to the author(s) and the published article's title, journal citation, and DOI. Open access publication funded by the Max Planck Society. photoexcitation circular dichroism [27,28], and highharmonic generation using bichromatic noncollinear lasers $[29,30]$.

Within this "zoo" of methods, PECD has distinguished itself as a particularly effective technique that leads to robust enantiosensitive chiral signals on the order of $1 \%-15 \%$ from variable targets, and which can also be applied to probe ultrafast chirality [18,31-33]. However, this technique is technically challenging because it requires measuring the angularly resolved photoelectron spectrum (PES). This fundamental constraint arises because chiral signals in standard PECD appear only as forwards/backwards asymmetries in the photoemission, whereas the angularly integrated PES is independent of the medium's handedness. An alternative technique that supports chiral dichroism in angularly integrated above-threshold ionization (ATI) [34,35], as well as in total ionization rates, would pave the way for simpler realizations for probing chirality and ultrafast dynamics.

Another closely related topic is optical chirality manipulation and the attempt to realize all-optical purification of chiral samples. This is a long sought-after goal that would ideally replace chemical-based approaches as a general method that is easier to implement and which would work consistently across molecular species. Unfortunately, it has proven to be extremely challenging. In the early 2000's a series of theoretical papers predicted that all-optical enantiopurification could be achieved by interacting a racemic mixture of chiral molecules with a sequence of resonantly tuned laser pulses [36-39]. This result has so far not been observed 
experimentally. Ultimately, similar to chirality characterization, all-optical enantioenrichment also requires strong dichroism to arise in the light-matter molecular response. In this respect, an optical setup giving rise to strong chiral discrimination in molecular ionization rates could also be utilized for enantioenrichment, as one could selectively photoionize a single molecular handedness to cleanse a racemic mixture.

Here we reformulate PECD with noncollinear and bichromatic laser pulses that are "locally chiral" [30,40]. The electric field generated by locally chiral light carries nonzero chirality density within the electric-dipole approximation, and is highly effective for enantioselectivity. We show that angularly resolved photoelectron spectra driven by locally chiral light leads to robust chiral signals of up to $15 \%$ that are no longer forwards/backwards asymmetric. Instead, dichroism arises in all hemispheres (i.e., forwards/backwards, up/down, left/right), and it is not purely symmetric or antisymmetric; that is, the resulting photoemission is in itself a chiral object. This fundamental aspect leads to chiral dichroism of up to $10 \%$ that survives angular integration in ATI spectra, and up to $5 \%$ in the total ionization rates. We demonstrate these effects and their generality through ab initio calculations in the chiral molecules: bromochlorofluoromethane, limonene, fenchone, and camphor. We also explore the correspondence between light's degree of chirality (DOC) [40] and the chiral-signal conversion efficiency, finding a strong correlation between the two. This result supports the use of the recently derived DOC as an effective measure to quantify chiral light-chiral matter interactions. This approach is expected to be highly useful for high-precision ultrafast enantiosensing. Most importantly, it can be directly applied to all-optical enantioseparation based on selective photodissociation, as we describe.

Method formulation. We begin by describing the optical beam configuration that leads to locally chiral light pulses, which employs the following $\omega$ - $2 \omega$ two-beam geometry:

$$
\mathrm{E}(t)=E_{0} A(t) \operatorname{Re}\left\{e^{i \omega t+i \eta} \hat{e}_{1}+\Delta e^{2 i \omega t} \hat{e}_{2}\right\},
$$

where $E_{0}$ is the $\omega$ electric field amplitude, $\Delta$ is the amplitude ratio between the beams, $\eta$ is a relative phase, $\hat{e}_{1,2}$ are unit vectors along the polarization direction of each beam (each beam is elliptical with ellipticities $\varepsilon_{1,2}$ and elliptical major axis angles $\beta_{1,2}$ w.r.t the $x$ axis), $A(t)$ is a dimensionless envelope function (taken in calculation to be trapezoidal with two-cycle rise and drop sections and a four-cycle flat top), and we have applied the dipole approximation neglecting spatial degrees of freedom. Equation (1) describes two noncollinear elliptically polarized laser beams of frequencies $\omega$ and $2 \omega$, respectively, which are focused together into a randomly oriented chiral medium (as illustrated in Fig. 1). Note that the field in Eq. (1) reduces to standard monochromatic circularly polarized light (CPL) upon substituting $\alpha=0, \Delta=0, \varepsilon_{1}=1$, which allows comparing chiral dichroism obtained from locally chiral light to the standard CPL regime.

The interaction of the laser field in Eq. (1) with chiral molecules is described here using time-dependent density functional theory (TDDFT) [41], in a real-space and realtime formulation using the OCTOPUS code [42-44]. This approach is extensively described in Refs. [42-44], and is a nonperturbative $a b$ initio method that in principle includes both electron-ion and electron-electron interactions. We del-

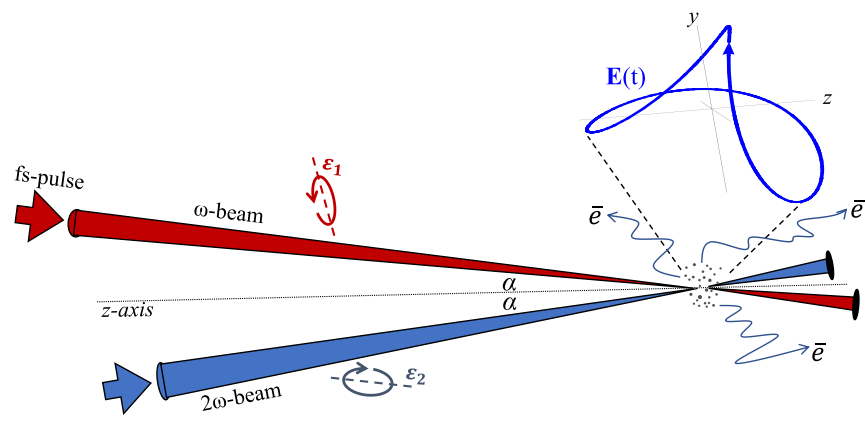

FIG. 1. Illustration of locally chiral laser field configuration that is generated by $\omega-2 \omega$ biochromatic noncollinear laser pulses. Here two beams of carrier frequencies $\omega$ and $2 \omega$ are focused into a gas of randomly oriented chiral molecules with an opening angle $2 \alpha$. Each beam is elliptical with ellipticities $\varepsilon_{1,2}$, and possibly different elliptical major axes $\left(\beta_{1,2}\right)$. The blue Lissajou represents the total electric field at the focus, which exhibits no improper rotational symmetries, rendering it "locally chiral." The strong-field photoionizes electrons that are measured in an angular- and momentum-resolved fashion.

egate technical details about the method to the Supplemental Material (SM) [45]. For simplicity, calculations in the main text employ the single active electron approximation (i.e., all deeper Kohn-Sham states are kept frozen during propagation), which has been proven very effective in PECD [21,24,25,46] (we explore the validity of this approximation against full TDDFT simulations in the SM [45]). To calculate the angularly resolved PES, we employ here the surface flux method $t$-SURFF [47-51]. Calculations are performed consecutively for varying molecular orientations of both enantiomers to obtain full orientation averaging (see SM for details [45]).

In order to put our scheme into perspective, we first reformulate the main physical observable of PECD, which is a chiral dichroism observed in the angularly resolved PES from mirror-image enantiomers. Theoretically, such a dichroism is obtained by subtracting the orientation-averaged PES calculated from both enantiomers that interact with the same CPL, and integrating along one axis (which experimentally occurs in velocity map imaging (VMI) [6]):

$$
\operatorname{PECD}\left(k_{x}, k_{z}\right)=\frac{P_{R}\left(k_{x}, k_{z}\right)-P_{S}\left(k_{x}, k_{z}\right)}{\max \left\{P_{R}\left(k_{x}, k_{z}\right)\right\}},
$$

where $P_{R / S}\left(k_{x}, k_{z}\right)$ is the momentum-resolved photoelectron distribution after integration along the transverse $y$ axis from $R / S$ enantiomers, respectively, and the $z$ axis is the propagation axis of the laser pulse. Note that we have normalized the PECD to the maximal power obtained in $P_{R}\left(k_{x}, k_{z}\right)$, which for the CPL case is identical for both enantiomers. This naturally limits PECD to have theoretical bounds from-200\% to $200 \%$. We also note that enantiomeric exchange in Eq. (2) $(R \rightarrow S)$ is equivalent to exchanging the helicity of the CPL, i.e., the chiral dichroism is equivalent to the circular dichroism. Importantly, Eq. (2) exhibits exact forwards/backwards asymmetry in the PECD; that is, upon exchanging the medium's handedness $(R \rightarrow S)$ one finds a similar response with an opposite sign along $k_{z}$ [6]. The physical origin of this effect is a symmetry exhibited by any collinear laser beam-the electric field is symmetric under the transformation $E_{\mathrm{z}} \rightarrow-E_{z}$ within 
the dipole approximation [52]. Major consequences of this asymmetry are (i) no discrimination is observed in the $x y$ plane (i.e., in up/down or left/right hemispheres), and (ii) no discrimination survives angular integration.

As opposed to the CPL case, we now formulate the main physical observables of photoelectron spectroscopy with locally chiral light. Here, one may define photoelectron chiral dichroism $(P E X D)$ with respect to any hemisphere (or Cartesian plane):

$$
\operatorname{PEXD}\left(k_{i}, k_{j}\right)=2 \frac{P_{R}\left(k_{i}, k_{j}\right)-P_{S}\left(k_{i}, k_{j}\right)}{\max \left\{P_{R}\left(k_{i}, k_{j}\right)\right\}+\max \left\{P_{S}\left(k_{i}, k_{j}\right)\right\}},
$$

where $i$ and $j$ denote Cartesian indices, and Eq. (3) is still bound from-200\% to $200 \%$, though now the maximal values of $P_{R}\left(k_{i}, k_{j}\right)$ and $P_{S}\left(k_{i}, k_{j}\right)$ in the denominator are not necessarily identical. This is a consequence of the light's local chirality, which breaks the forwards/backwards asymmetry. In fact, following the structure of the light field [30,40], the resulting function $\operatorname{PEXD}\left(k_{i}, k_{j}\right)$ does not exhibit any particular symmetry relation-it contains both symmetric and antisymmetric parts with respect to spatial reflections or enantiomeric exchange. It is also important to point out that here the chiral dichroism is no longer equivalent to circular dichroism due to the superposition structure of the field in Eq. (1), though one may still generate an equivalent notion to circular dichroism by reflecting the experimental setup between both beams. From this point on we refer to the chiral dichroism as the main observable.

Due to the chiral nature of the PES, it is also appropriate to discuss the chiral dichroism of the full photoelectron distribution:

$$
P E X D(\mathbf{k})=2 \frac{P_{R}(\mathbf{k})-P_{S}(\mathbf{k})}{\max \left\{P_{R}(\mathbf{k})\right\}+\max \left\{P_{S}(\mathbf{k})\right\}},
$$

where $\mathbf{k}$ is the three-dimensional outgoing photoelectron momentum, and $P_{R}(\mathbf{k})$ contains the full PES. It is notable that for the CPL case, Eq. (4) still only leads to chiral dichroism due to an exchange $k_{z} \rightarrow-k_{z}$, where for locally chiral light any possible exchange of momentum could lead to chiral dichroism. While the object in Eq. (4) is very difficult to experimentally resolve, it can be much more revealing towards the possible enantioselectivity potential of $P E X D$. Particularly, one can take the maximal value of the chiral dichroism as a measure for the selectivity: $P E X D_{\max }=\max \{P E X D(k)\}$. We utilize this quantity in order to compare between the chiral responses in different conditions, and from different molecules.

Lastly, two more vital quantities should be defined. First, since locally chiral light breaks all symmetry relations for photoemission between enantiomers, we can expect chiral dichroism to survive angular integration:

$$
A T I_{\mathrm{X} D}(\varepsilon)=2 \frac{A T I_{R}(\varepsilon)-A T I_{S}(\varepsilon)}{\max \left\{A T I_{R}(\varepsilon)\right\}+\max \left\{A T I_{S}(\varepsilon)\right\}},
$$

where $A T I_{\mathrm{X} D}(\varepsilon)$ is the dichroism obtained at the photoelectron energy $\varepsilon$, and $A T I_{R / S}(\varepsilon)$ are the individual ATI spectra from each enantiomer. For simplicity, we have normalized Eq. (5) by the maximal photoelectron energy-resolved yield, which gives a good estimate to the size of the discrimination. Alternatively, one may normalize the ATI chiral dichroism per each peak, as is done for instance in high-harmonic genera- tion [29,30,53-55], which can lead to overall larger values. Second, by integrating over the energy in Eq. (5) we obtain the discrimination in total photoelectron yield:

$$
I_{\mathrm{X} D}=2 \frac{I_{R}-I_{S}}{I_{R}+I_{S}} .
$$

Here $I_{\mathrm{X} D}$ is a pseudo scalar that is normalized from-200\% to $200 \%$ that indicates the total excess electrons ionized from one enantiomer compared to the other. We emphasize that both $A T I_{\mathrm{X} D}(\varepsilon)$ and $I_{\mathrm{X} D}$ are strictly zero for CPL. In fact, they are also strictly zero for more complex bichromatic fields $[24,25]$, or for any light that does not possess local chirality.

Numerical results. Having analytically formulated the main physical observables for $P E X D$, we turn to practical calculations. We begin our analysis with the smallest stable chiral molecule, Bromochlorofluoromethane $(\mathrm{CBrClFH})$. We calculate the PES from $R$ - and $S$-CBrClFH driven by the locally chiral field in Eq. (1) (for numerical details see the SM [45]). The optical beam parameters are chosen according to a maximally chiral configuration that was predicted in Ref. [40] to maximize light's DOC, which is intuitively expected to yield relatively large chiral signals. Figures 2(a)-2(c) show the resulting $P E X D$ in all Cartesian planes [according to Eq. (3)], which exhibits strong dichroism in all hemispheres and reaches a maximal value of $11.51 \%$ (this can be compared to a value of $2.01 \%$ obtained from CPL in similar conditions with $800 \mathrm{~nm}$ light in the strong-field regime, or $5.05 \%$ in the perturbative regime in the low-energy part of the spectrum with $400 \mathrm{~nm}$ light; see SM for details [45]). A striking feature here is that Figs. 2(a)-2(c) exhibit no symmetry relations; i.e., the standard forwards/backwards asymmetry of PECD is broken. This is a direct consequence of the use of locally chiral light, which breaks the mirror-image relation between enantiomers within the electric-dipole approximation. It is worth mentioning that for this symmetry breaking to occur, pathways for photoemission must mix photons from both of the noncollinear beams that comprise the locally chiral field. The origin of the effect is owing to the fact that the fieldmixing terms generate an asymmetry that is present in all tensorial coefficients of the Legendre polynomial expansion of the PES [20]. This includes the zero-order coefficient $b_{0}$ that does not contribute to standard PECD (and which can survive orientation averaging), as well as all odd and even terms (whereas usually only odd terms contribute to the chiral signal).

Figure 3 presents the angularly integrated ATI spectra from both enantiomers, and the resulting ATI chiral dichroism according to Eq. (5). Dichroism of up to $4.29 \%$ is obtained for the low-energy ATI peaks, and dichroism of up to $2 \%$ survives up to $7 \mathrm{eV}$. In the SM [45] we present calculations at equivalent conditions but higher laser powers, where ATI chiral dichroism of up to $7 \%$ is obtained; i.e., stronger field amplitudes generally increase the ATI dichroism, as expected (since the mixing between fields is more prominent). Note, though, that this can also cause the ATI chiral dichroism to strongly oscillate from peak to peak (see discussion in SM [45]). Overall, this broad energy range and strong signal can be highly useful for chiral spectroscopy.

Additional integration of the ATI spectra from both enantiomers leads to a total photoelectron yield chiral dichroism 

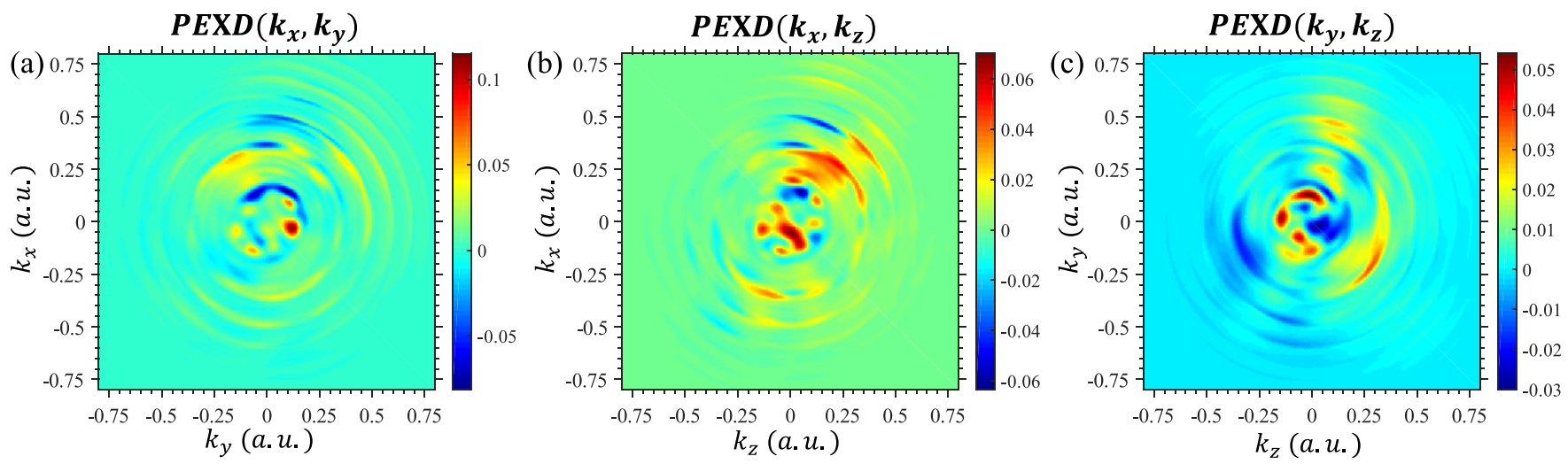

FIG. 2. $P E X D$ using locally chiral light from $\mathrm{CBrClFH}$. (a-c) $P E X D\left(k_{i}, k_{j}\right)$ where $i, j$ are Cartesian indices. Electron momenta are given in atomic units. The locally chiral $\omega$-2 $\omega$ laser beam setup used is the one predicted in Ref. [40] to carry maximal DOC, where the parameters in Eq. (1) are set to: $\lambda=800 \mathrm{~nm}(\omega=1.55 \mathrm{eV}), \varepsilon_{1}=-0.37, \varepsilon_{2}=0.58, \beta_{1}=23.9^{\circ}, \beta_{2}=28.7^{\circ}, \alpha=30.1^{\circ}, \Delta=0.77, \eta=0.4$, and $I_{0}=$ $2 \times 10^{13} \mathrm{~W} / \mathrm{cm}^{2}$. Note that the color scales are not identical in all subplots and range from the minimal to maximal values of dichroism in each case.

[according to Eq. (6)] of $2.46 \%$. That is, when $R$ - $\mathrm{CBrClFH}$ interacts with the strong field in Eq. (1), electrons are photoionized at a rate that is $2.46 \%$ faster than those from $S$-CBrClFH. This result is somewhat unintuitive, since both molecules are mirror images of one another, and since the randomly oriented media are fully isotropic. Still, the lack of inversion symmetry in the randomly oriented medium, accompanied by the lack of such symmetry in the optical setup [52], allows one of the enantiomers to interact more efficiently with the light compared to the other enantiomer. Notably, such an approach can still be applied to time-resolved spectroscopy of dynamical processes, since the laser pulses have femtosecond durations [the full width at half maximum (FWHM) in our calculations is $\sim 16 \mathrm{fs}]$.

So far, we have explored a single laser beam geometry. For practical applications it is important to analyze the chiral dichroism for varying beam configurations to optimize the signal. Hence, we explore the beam parameter space in the context of the efficiency of the chiral light-matter response, and scan the opening angle $\alpha$, and amplitude ratio $\Delta$, while calculating the total ionization rate discrimination. In Ref. [40], the seven degrees of freedom that characterize the field in Eq. (1) (e.g., ellipticities, phases, amplitudes, etc.) were optimized to yield a maximal value for its DOC, which is a theoretical measure that quantifies the extent of symmetry breaking expected by this light [30,40]. It is crucial to determine if the DOC indeed correlates to the obtained chiral signals if it is to be used for applications, which has not yet been established (i.e., as is known for the ellipticity of light in the standard case of monochromatic CPL). Results are presented in Figs. 4(a) and 4(b), where in each scan all other beam parameters are fixed to the maximal DOC configuration. Figure 4(a) shows a strong increase of the total yield dichroism vs the opening angle, which is in perfect correspondence with the increase of light's DOC vs $\alpha$ [Fig. 4(c)]. Similarly, Fig. 4(b) shows a strong increase in the discrimination up to beam amplitude ratios of $\Delta=0.6$, where the signal maximizes at $3.09 \%$. This increase agrees with the increase in light's DOC vs $\Delta$ [Fig. 4(c)], though here there is a slight discrepancy since light's DOC maximizes at $\Delta=0.77$, while the chiral response maximizes at $\Delta=0.6$. Overall, these results support a significant correspondence between the DOC of the laser field and the chiral-signal conversion efficiency [see trend line in Fig. 4(c) with $R^{2}=0.98$ ], meaning that the DOC is a useful
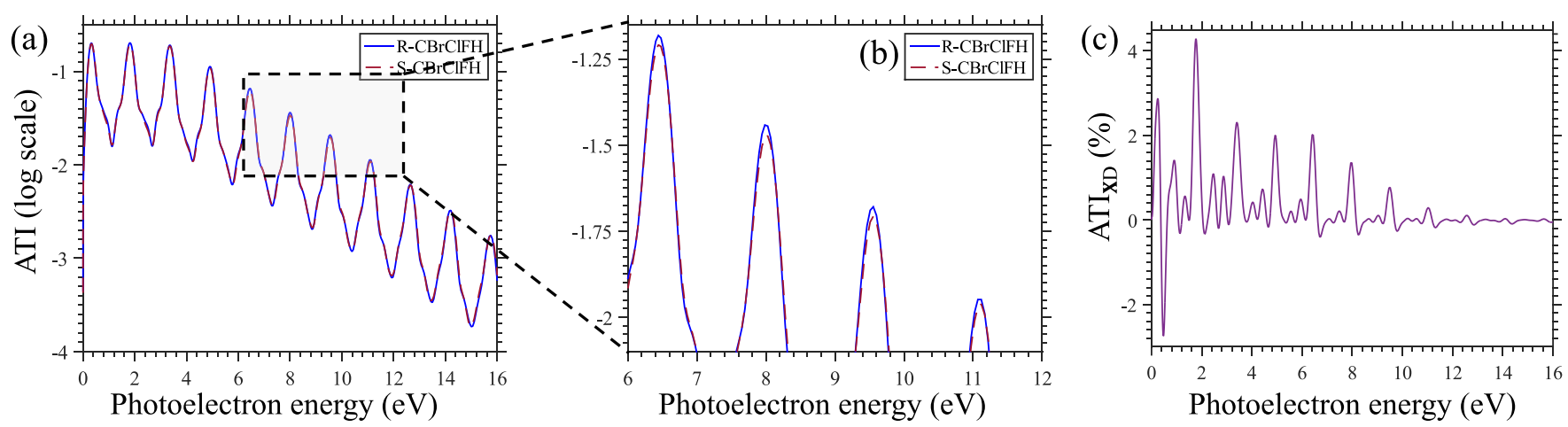

FIG. 3. ATI chiral dichroism using locally chiral light from CBrClFH. (a) ATI spectra from $R$ - and $S$-CBrClFH. Inset in (b) shows magnification around the 6-12 eV region, clearly showing a discrimination in the photoelectron yield around the ATI peaks. (c) Resulting ATI chiral dichroism using Eq. (5), normalized to the maximal ATI power. The locally chiral $\omega$ - $2 \omega$ laser beam setup is identical to that in Fig. 2. 

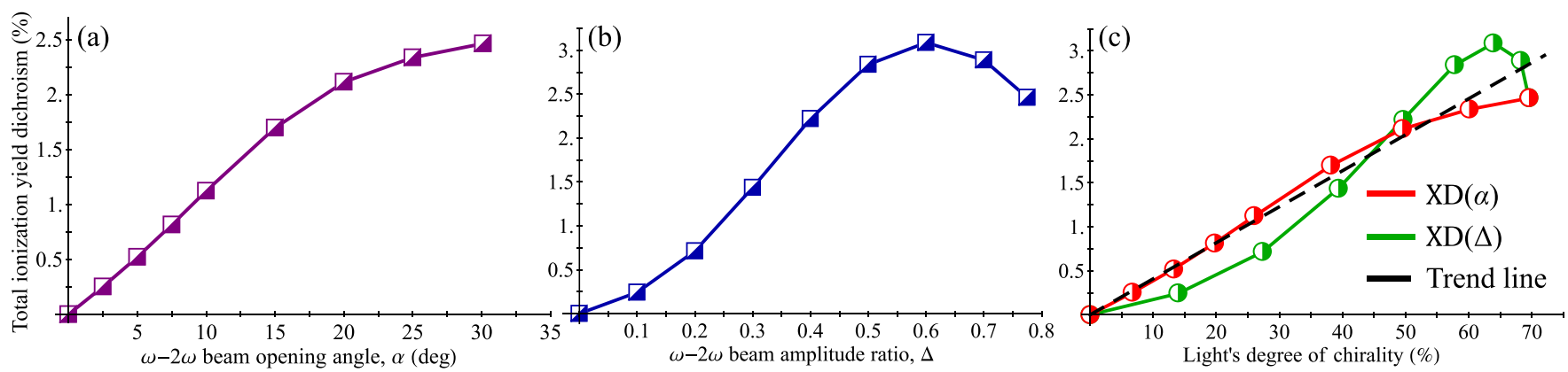

FIG. 4. Physical behavior of ionization rate chiral dichroism (XD) from locally chiral light in CBrClFH vs laser beam parameters, and correspondence with light's DOC. (a) Total photoelectron yield chiral dichroism vs $\omega$ - $2 \omega$ laser beam opening angle, $\alpha$. (b) Same as (a), but as a function of the $\omega-2 \omega$ beam amplitude ratios, $\Delta$. (c) Correspondence between total photoelectron yields chiral dichroism in (a,b), and light's DOC, as $\alpha$ and $\Delta$ are varied (trend line shows linear regression to all data with $R^{2}=0.98$ ). In all calculations the laser beam parameters are identical to those in Figs. 2 and 3, but where only $\alpha$ or $\Delta$ are varied. DOC calculations are performed following the prescriptions in Ref. [40].

measure for chiral light-chiral matter interaction strengths and can be formally used to predict field geometries for chiral spectroscopy or enantioseparation.

Another important point that arises from Fig. 4 is that strong ionization rate dichroism is maintained even for small beam opening angles. For instance, it remains at $0.25 \%$ for $\alpha=2.5^{\circ}$. While this value may seem small compared to the maximally obtained value of $3 \%$, it is still orders of magnitude larger than chiral signals from standard chiroptical techniques. Moreover, the field parameters were optimized in Ref. [40] for the large opening angle case; thus, one can expect even larger values after optimization. Overall, the scheme is also applicable in experimental setups with few-degree beam opening angles.

At this point we note that throughout our analysis we have neglected the spatial dependence of the electromagnetic field (e.g., focal averaging and other macroscopic effects). This approximation is not fully valid for the noncollinear configuration since the phase of the electromagnetic field can vary in space. This in turn can alter the DOC of light and its sign across the interaction region, which might affect chiral signals. To avoid this effect, one can choose the driving field geometry such that the phase of the field has a constant sign in space as is demonstrated in Ref. [30], rendering the microscopic predictions valid. Alternatively, one may work in the IR regime with small beam opening angles such that the phase remains approximately constant in a narrow interaction region. We found that in this regime (with a fundamental wavelength of $1600 \mathrm{~nm}$ at $\alpha=2.5^{\circ}$ and stronger laser powers of $5 \times 10^{13} \mathrm{~W} / \mathrm{cm}^{2}$ ) one still obtains $0.41 \%$ chiral dichroism in the total molecular ionization rates.

Having established the main results in $\mathrm{CBrClFH}$, we demonstrate the generality of the technique by performing calculations in three more benchmark chiral molecules: limonene, fenchone, and camphor. Figures 5(a)-5(c) present the corresponding ATI chiral dichroism from each species in similar settings to those in Fig. 3 (see SM [45] for the corresponding PEXD). Strong discrimination is observed in ATI peaks reaching as high as $10 \mathrm{eV}$ from all species, where limonene shows the strongest response with a maximal value of $10.79 \%$. The total photoelectron yield from each species also demonstrates large dichroisms of up to $5 \%$. Table I summarizes the different chiral observables calculated for these molecules in identical settings, as well as in the strong-field CPL case in $\mathrm{CBrClFH}$.

All-optical chiral purification. Lastly, we discuss how our approach can be used for chiral purification and detail a possible experimental setup. Unlike previous proposals that suggested converting one enantiomer to another or spatially separating enantiomers by a complex sequence of fine-tuned (resonant) optical pulses [36-39], we here propose a different path. By using the setup described above, a strong chiral dichroism arises in the total molecular ionization cross sections. Therefore, irradiating a racemic mixture will lead
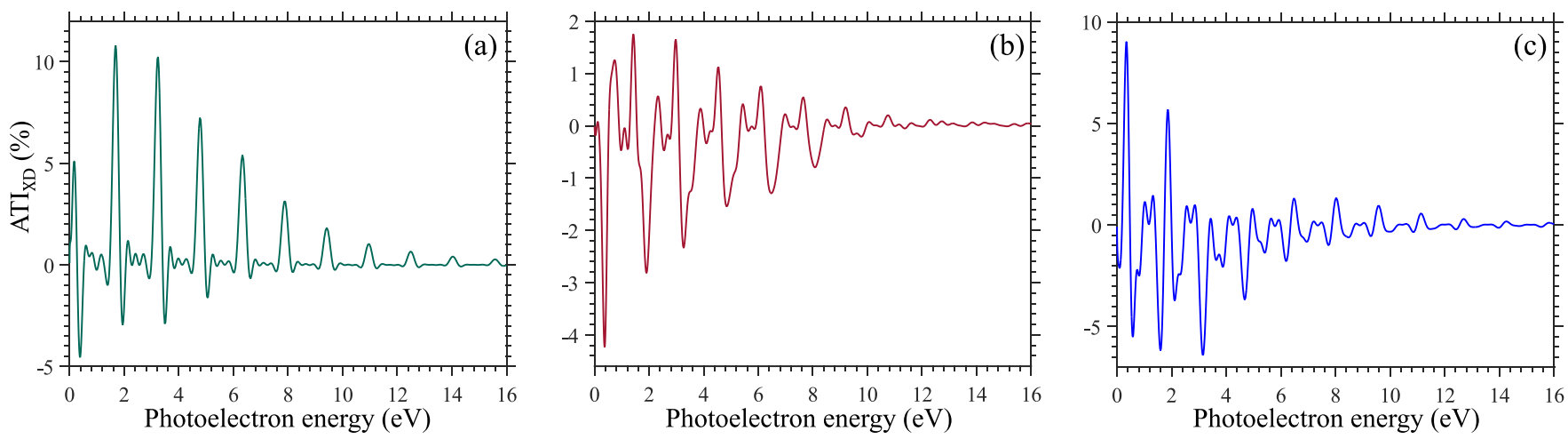

FIG. 5. ATI chiral dichroism using locally chiral light from: (a) limonene, (b) fenchone, and (c) camphor, respectively. Calculations are performed in $\omega-2 \omega$ optical beam settings similar to those in Figs. 2 and 3. 
TABLE I. Summary of calculated chiral signals obtained from different chiral molecules and within different enantiosensitive measures as described in the text. All cases utilize the same optical setup as in Figs. 2, 3, and 5, except for the CPL case, which has the same total laser power $\left(I_{0}=4 \times 10^{13} \mathrm{~W} / \mathrm{cm}^{2}\right)$. All values are in absolute sizes (signs have been removed).

\begin{tabular}{|c|c|c|c|c|c|}
\hline & $\mathrm{CBrClFH}-\mathrm{CPL}(800 \mathrm{~nm})$ & $\mathrm{CBrClFH}$ & Limonene & Fenchone & Camphor \\
\hline $\max \left\{P E X D_{i j}\right\}$ & $2.01 \%$ & $11.51 \%$ & $13.61 \%$ & $12.08 \%$ & $10.89 \%$ \\
\hline$P E X D_{\max }$ & $5.91 \%$ & $14.38 \%$ & $37.41 \%$ & $24.12 \%$ & $24.85 \%$ \\
\hline $\max \left\{A T I_{\mathrm{X} D}\right\}$ & 0 & $4.29 \%$ & $10.79 \%$ & $4.24 \%$ & $9.01 \%$ \\
\hline$I_{\mathrm{X} D}$ & 0 & $2.46 \%$ & $4.81 \%$ & $1.82 \%$ & $1.95 \%$ \\
\hline
\end{tabular}

to selective ionization of one molecular handedness over the other. Upon photoionization, the molecules dissociate to smaller fragments that can be cleansed by various techniques (e.g., chromatography or membranes [56]). This approach can be applied recursively in a closed-loop over time to enrich chiral samples. This route takes advantage of our approach being applicable to both liquid and gas phases, and to all chiral molecules without needing to fine-tune any of the laser parameters. From a practical point of view of conversion efficiency, it is essential that the ionization chiral dichroism is a relatively large number on the order of several percent as expected with our approach (previously available chiral dichroisms on the order of $10^{-4} \%-10^{-5} \%$ from approaches based on magnetic-dipole transitions in the optical range are impractical).

Discussion and outlook. To summarize, we have reformulated the method of PECD to the use of noncollinear and bichromatic laser pulses that are locally chiral $[30,40]$. We theoretically derived the main chirality-sensitive observables for this approach, and performed $a b$ initio calculations on a set of four chiral molecules to verify its validity. The use of locally chiral light is shown to break the forwards/backwards asymmetry of standard PECD leading to several attractive physical properties: (i) a strong chiral dichroism in the PES is observed in all hemispheres (i.e., in all Cartesian planes), and the photoemission is a chiral entity that does not exhibit any symmetry relation upon enantiomeric exchange. (ii) ATI dichroism of up to $10 \%$ is obtained in the angularly integrated photoelectron spectra. (iii) The total ionization rate (angularly and energy integrated) from the orientation-averaged chiral media exhibits a large chiral dichroism of $\sim 5 \%$ (note that this is on the same order of magnitude as chiral dichroisms that are standardly obtained in fully angularly resolved PECD; see, e.g., results in Refs. $[17,19,20,23,25,33]$ ). (iv) The method is independent of the femtosecond duration of the driving pulse, such that it can still be applied for exploring ultrafast dynamics. These results are qualitatively upheld also beyond the single active electron approximation as we have shown with full TDDFT calculations that include dynamical correlations (see SM [45]). Furthermore, we have established that there is a strong correlation between light's degree of chirality and the chiral-signal conversion efficiency, suggesting that it can be utilized in future studies to predict ideal laser beam setups for chiral spectroscopy and manipulation.

The exciting prospects of this approach pave the way for using ATI and photoionization measurements for chiral spectroscopy. The enhanced sensitivity also means that the method is likely suitable to probe other static or dynamical properties of molecules, including valence structure and dynamical correlations (see discussion in SM [45]). Notably, this technique can also be extended to explore chiral solids, and in particular, chiral topological effects in quantum materials. We have outlined how the large discrimination in the total molecular ionization rates can pave the way to efficient all-optical enantioseparation via selective photoionization. Looking forward, our work will advance ultrafast chirality spectroscopy and manipulation, and especially, motivate the use of locally chiral light for the enhancement of existing techniques.

Acknowledgments. We thank Shaked Rozen from Weisman Institute, Israel, and Bernard Pons from Université Bordeaux, France, for helpful discussions. We acknowledge financial support from the European Research Council (Grant No. ERC-2015-AdG-694097), the Cluster of Excellence 'CUI: Advanced Imaging of Matter' of the Deutsche Forschungsgemeinschaft (DFG) - EXC 2056 - project ID 390715994, Grupos Consolidados (IT1249-19), partially supported by the Federal Ministry of Education and Research Grant RouTe13N14839, the SFB925 "Light induced dynamics and control of correlated quantum systems". The Flatiron Institute is a division of the Simons Foundation. O.N. gratefully acknowledges the support of the Adams Fellowship Program of the Israel Academy of Sciences and Humanities and support from the Alexander von Humboldt foundation.
[1] H. Rhee, Y.-G. June, J.-S. Lee, K.-K. Lee, J.-H. Ha, Z. H. Kim, S.-J. Jeon, and M. Cho, Femtosecond characterization of vibrational optical activity of chiral molecules, Nature 458, 310 (2009).

[2] K. Higashijima, Dynamical chiral-symmetry breaking, Phys. Rev. D 29, 1228 (1984).

[3] P. Hosur, S. Ryu, and A. Vishwanath, Chiral topological insulators, superconductors, and other competing or- ders in three dimensions, Phys. Rev. B 81, 045120 (2010).

[4] S.-T. Wang, D.-L. Deng, and L.-M. Duan, Probe of ThreeDimensional Chiral Topological Insulators in an Optical Lattice, Phys. Rev. Lett. 113, 033002 (2014).

[5] W. H Brooks, W. C Guida, and K. G Daniel, The significance of chirality in drug design and development, Curr. Top. Med. Chem. 11, 760 (2011). 
[6] N. Berova, P. L. Polavarapu, K. Nakanishi, and R. W. Woody, Comprehensive Chiroptical Spectroscopy: Applications in Stereochemical Analysis of Synthetic Compounds, Natural Products, and Biomolecules (John Wiley \& Sons, New York, 2012), Vol. 2.

[7] P. Fischer, D. S. Wiersma, R. Righini, B. Champagne, and A. D. Buckingham, Three-Wave Mixing in Chiral Liquids, Phys. Rev. Lett. 85, 4253 (2000).

[8] G. J. Simpson, Molecular origins of the remarkable chiral sensitivity of second-order nonlinear optics, Chem. Phys. Chem. 5, 1301 (2004).

[9] M. A. Belkin and Y. R. Shen, Non-linear optical spectroscopy as a novel probe for molecular chirality, Int. Rev. Phys. Chem. 24, 257 (2005).

[10] D. Patterson, M. Schnell, and J. M. Doyle, Enantiomer-specific detection of chiral molecules via microwave spectroscopy, Nature 497, 475 (2013).

[11] S. Eibenberger, J. Doyle, and D. Patterson, Enantiomer-Specific State Transfer of Chiral Molecules, Phys. Rev. Lett. 118, 123002 (2017).

[12] M. Pitzer, M. Kunitski, A. S. Johnson, T. Jahnke, H. Sann, F. Sturm, L. P. H. Schmidt, H. Schmidt-Böcking, R. Dörner, J. Stohner, J. Kiedrowski, M. Reggelin, S. Marquardt, A. Schießer, R. Berger, and M. S. Schöffler, Direct determination of absolute molecular stereochemistry in gas phase by Coulomb explosion imaging, Science 341, 1096 (2013).

[13] P. Herwig, K. Zawatzky, M. Grieser, O. Heber, B. JordonThaden, C. Krantz, O. Novotný, R. Repnow, V. Schurig, D. Schwalm, Z. Vager, A. Wolf, O. Trapp, and H. Kreckel, Imaging the absolute configuration of a chiral epoxide in the gas phase, Science 342, 1084 (2013).

[14] C. Pérez, A. L. Steber, S. R. Domingos, A. Krin, D. Schmitz, and M. Schnell, Coherent enantiomer-selective population enrichment using tailored microwave fields, Angew. Chem., Int. Ed. 56, 12512 (2017).

[15] B. Ritchie, Theory of the angular distribution of photoelectrons ejected from optically active molecules and molecular negative ions, Phys. Rev. A 13, 1411 (1976).

[16] I. Powis, Photoelectron circular dichroism of the randomly oriented chiral molecules glyceraldehyde and lactic acid, J. Chem. Phys. 112, 301 (1999).

[17] N. Böwering, T. Lischke, B. Schmidtke, N. Müller, T. Khalil, and U. Heinzmann, Asymmetry in Photoelectron Emission from Chiral Molecules Induced by Circularly Polarized Light, Phys. Rev. Lett. 86, 1187 (2001).

[18] A. Comby, S. Beaulieu, M. Boggio-Pasqua, D. Descamps, F. Légaré, L. Nahon, S. Petit, B. Pons, B. Fabre, Y. Mairesse, and V. Blanchet, Relaxation dynamics in photoexcited chiral molecules studied by time-resolved photoelectron circular dichroism: Toward chiral femtochemistry, J. Phys. Chem. Lett. 7, 4514 (2016).

[19] C. Lux, M. Wollenhaupt, T. Bolze, Q. Liang, J. Köhler, C. Sarpe, and T. Baumert, Circular dichroism in the photoelectron angular distributions of camphor and fenchone from multiphoton ionization with femtosecond laser pulses, Angew. Chem., Int. Ed. 51, 5001 (2012).

[20] C. S. Lehmann, N. B. Ram, I. Powis, and M. H. M. Janssen, Imaging photoelectron circular dichroism of chiral molecules by femtosecond multiphoton coincidence detection, J. Chem. Phys. 139, 234307 (2013).
[21] A. N. Artemyev, A. D. Müller, D. Hochstuhl, and P. V. Demekhin, Photoelectron circular dichroism in the multiphoton ionization by short laser pulses. I. Propagation of single-activeelectron wave packets in chiral pseudo-potentials, J. Chem. Phys. 142, 244105 (2015).

[22] S. Beaulieu, A. Ferré, R. Géneaux, R. Canonge, D. Descamps, B. Fabre, N. Fedorov, F. Légaré, S. Petit, T. Ruchon, V. Blanchet, Y. Mairesse, and B. Pons, Universality of photoelectron circular dichroism in the photoionization of chiral molecules, New J. Phys. 18, 102002 (2016).

[23] A. D. Müller, E. Kutscher, A. N. Artemyev, and P. V. Demekhin, Photoelectron circular dichroism in the multiphoton ionization by short laser pulses. III. Photoionization of fenchone in different regimes, J. Chem. Phys. 152, 044302 (2020).

[24] P. V Demekhin, A. N. Artemyev, A. Kastner, and T. Baumert, Photoelectron Circular Dichroism with Two Overlapping Laser Pulses of Carrier Frequencies $\omega$ and $2 \omega$ Linearly Polarized in Two Mutually Orthogonal Directions, Phys. Rev. Lett. 121, 253201 (2018).

[25] S. Rozen, A. Comby, E. Bloch, S. Beauvarlet, D. Descamps, B. Fabre, S. Petit, V. Blanchet, B. Pons, N. Dudovich, and Y. Mairesse, Controlling Subcycle Optical Chirality in the Photoionization of Chiral Molecules, Phys. Rev. X 9, 031004 (2019).

[26] K. Fehre, S. Eckart, M. Kunitski, C. Janke, D. Trabert, M Hofmann, J. Rist, M. Weller, A. Hartung, L. P. H. Schmidt, T. Jahnke, H. Braun, T. Baumert, J. Stohner, P. V. Demekhin, M. S. Schöffler, and R. Dörner, Strong Differential Photoion Circular Dichroism in Strong-Field Ionization of Chiral Molecules, Phys. Rev. Lett. 126, 083201 (2021).

[27] S. Beaulieu, A. Comby, D. Descamps, B. Fabre, G. A. Garcia, R. Géneaux, A. G. Harvey, F. Légaré, Z. Mašín, and L. Nahon, Photoexcitation circular dichroism in chiral molecules, Nat. Phys. 14, 484 (2018).

[28] A. G. Harvey, Z. Mašín, and O. Smirnova, General theory of photoexcitation induced photoelectron circular dichroism, J. Chem. Phys. 149, 064104 (2018).

[29] O. Neufeld, D. Ayuso, P. Decleva, M. Y. Ivanov, O. Smirnova, and O. Cohen, Ultrasensitive Chiral Spectroscopy by Dynamical Symmetry Breaking in High Harmonic Generation, Phys. Rev. X 9, 031002 (2019).

[30] D. Ayuso, O. Neufeld, A. F. Ordonez, P. Decleva, G. Lerner, O. Cohen, M. Ivanov, and O. Smirnova, Synthetic chiral light for efficient control of chiral light-matter interaction, Nat. Photonics 13, 866 (2019).

[31] S. Beaulieu, A. Comby, B. Fabre, D. Descamps, A. Ferre, G. Garcia, R. Geneaux, F. Legare, L. Nahon, S. Petit, T. Ruchon, B. Pons, V. Blanchet, and Y. Mairesse, Probing ultrafast dynamics of chiral molecules using time-resolved photoelectron circular dichroism, Faraday Discuss. 194, 325 (2016)

[32] R. E. Goetz, T. A. Isaev, B. Nikoobakht, R. Berger, and C. P. Koch, Theoretical description of circular dichroism in photoelectron angular distributions of randomly oriented chiral molecules after multi-photon photoionization, J. Chem. Phys. 146, 024306 (2017).

[33] S. Beaulieu, A. Comby, A. Clergerie, J. Caillat, D. Descamps, N. Dudovich, B. Fabre, R. Géneaux, F. Légaré, S. Petit, B. Pons, G. Porat, T. Ruchon, R. Taïeb, V. Blanchet, and Y. Mairesse, Attosecond-resolved photoionization of chiral molecules, Science 358, 1288 (2017). 
[34] D. B. Milošević, G. G. Paulus, D. Bauer, and W. Becker, Abovethreshold ionization by few-cycle pulses, J. Phys. B: At., Mol. Opt. Phys. 39, R203 (2006).

[35] T. Schultz and M. Vrakking, Attosecond and XUV Physics: Ultrafast Dynamics and Spectroscopy (Wiley, New York, 2014).

[36] P. Král, I. Thanopulos, M. Shapiro, and D. Cohen, Two-Step Enantio-Selective Optical Switch, Phys. Rev. Lett. 90, 033001 (2003).

[37] D. Gerbasi, P. Brumer, I. Thanopulos, P. Král, and M. Shapiro, Theory of the two step enantiomeric purification of 1,3 dimethylallene, J. Chem. Phys. 120, 11557 (2004).

[38] D. Gerbasi, M. Shapiro, and P. Brumer, Theory of "laser distillation" of enantiomers: Purification of a racemic mixture of randomly oriented dimethylallene in a collisional environment, J. Chem. Phys. 124, 074315 (2006).

[39] X. Li and M. Shapiro, Theory of the optical spatial separation of racemic mixtures of chiral molecules, J. Chem. Phys. 132, 194315 (2010).

[40] O. Neufeld, M. E. Tzur, and O. Cohen, Degree of chirality of electromagnetic fields and maximally chiral light, Phys. Rev. A 101, 053831 (2020).

[41] M. A. L. Marques, C. A. Ullrich, F. Nogueira, A. Rubio, K. Burke, and E. K. U. Gross, Time-dependent density functional theory, in Time-Dependent Density Functional Theory, edited by M. A. L. Marques, C. Ullrich, F. Nogueira, A. Rubio, K. Burke, and E. K. U. Gross (Springer, Berlin, Heidelberg, 2003).

[42] A. Castro, H. Appel, M. Oliveira, C. A. Rozzi, X. Andrade, F. Lorenzen, M. A. L. Marques, E. K. U. Gross, and A. Rubio, OCTOPUS: A tool for the application of time-dependent density functional theory, Phys. Status Solidi 243, 2465 (2006).

[43] X. Andrade, D. Strubbe, U. De Giovannini, H. Larsen, M. J. T. Oliveira, J. Alberdi-rodriguez, A. Varas, I. Theophilou, N. Helbig, M. J. Verstraete, L. Stella, F. Nogueira, A. Castro, M. A. L. Marques, and A. Rubio, Real-space grids and the octopUs code as tools for the development of new simulation approaches for electronic systems, Phys. Chem. Chem. Phys. 17, 31371 (2015).

[44] N. Tancogne-Dejean, M. J. T. Oliveira, X. Andrade, H. Appel, C. H. Borca, G. Le Breton, F. Buchholz, A. Castro, S. Corni, A. A. Correa, U. De Giovannini, A. Delgado, F. G. Eich, J. Flick, G. Gil, A. Gomez, N. Helbig, H. Hübener, R. Jestädt, J. Jornet-Somoza et al., OCTOPUS, a computational framework for exploring light-driven phenomena and quantum dynamics in extended and finite systems, J. Chem. Phys. 152, 124119 (2020).

[45] See Supplemental Material at http://link.aps.org/supplemental/ 10.1103/PhysRevResearch.3.L032006 for the details about methodology used in the main text, as well as some additional results.

[46] I. Dreissigacker and M. Lein, Photoelectron circular dichroism of chiral molecules studied with a continuum-state-corrected strong-field approximation, Phys. Rev. A 89, 053406 (2014).

[47] L. Tao and A. Scrinzi, Photo-electron momentum spectra from minimal volumes: The time-dependent surface flux method, New J. Phys. 14, 013021 (2012).

[48] A. Scrinzi, $t$-SURFF: Fully differential two-electron photoemission spectra, New J. Phys. 14, 085008 (2012).

[49] P. Wopperer, U. De Giovannini, and A. Rubio, Efficient and accurate modeling of electron photoemission in nanostructures with TDDFT, Eur. Phys. J. B 90, 51 (2017).

[50] U. De Giovannini, H. Hübener, and A. Rubio, A firstprinciples time-dependent density functional theory framework for spin and time-resolved angular-resolved photoelectron spectroscopy in periodic systems, J. Chem. Theory Comput. 13, 265 (2017).

[51] S. A. Sato, H. Hübener, A. Rubio, and U. De Giovannini, First-principles simulations for attosecond photoelectron spectroscopy based on time-dependent density functional theory, Eur. Phys. J. B 91, 126 (2018).

[52] O. Neufeld, D. Podolsky, and O. Cohen, Floquet group theory and its application to selection rules in harmonic generation, Nat. Commun. 10, 405 (2019).

[53] R. Cireasa, A. E. Boguslavskiy, B. Pons, M. C. H. Wong, D. Descamps, S. Petit, H. Ruf, N. Thiré, A. Ferré, J. Suarez, J. Higuet, B. E. Schmidt, A. F. Alharbi, F. Légaré, V. Blanchet, B. Fabre, S. Patchkovskii, O. Smirnova, Y. Mairesse, V. R. Bhardwaj et al., Probing molecular chirality on a subfemtosecond timescale, Nat. Phys. 11, 654 (2015).

[54] D. Baykusheva and H. J. Wörner, Chiral Discrimination through Bielliptical High-Harmonic Spectroscopy, Phys. Rev. X 8, 031060 (2018).

[55] D. Baykusheva, D. Zindel, V. Svoboda, E. Bommeli, M. Ochsner, A. Tehlar, and H. J. Wörner, Real-time probing of chirality during a chemical reaction, Proc. Natl. Acad. Sci. U.S.A. 116, 23923 (2019).

[56] S. Fanali, P. R. Haddad, C. F. Poole, and M.-L. B. T. Riekkola, Liquid Chromatography Fundamentals and Instrumentation, 2nd ed. (Elsevier, Amsterdam, 2017). 